\title{
CaloCube: a novel calorimeter for high-energy cosmic rays in space
}
A. Rappoldi ${ }^{1, a}$, P.W.
Cattaneo $^{1, b}$
O. Adriani ${ }^{2,3}$, A. Agnesi ${ }^{1,4}$,
S. Albergo $0^{5,6}$,
L. Auditore ${ }^{7,8}$,
A. Basti ${ }^{10}$, E. Berti ${ }^{2,3}$, G. Bigongiari ${ }^{9,10}$, L. Bonechi ${ }^{2,3}$, S. Bonechi ${ }^{9,10}$, M. Bongi ${ }^{2,3}$, V. Bonvicini ${ }^{11}$, S. Bottai ${ }^{3}$, P. Brogi $i^{9,10}$, G. Cappello ${ }^{6}$, G. Carotenuto ${ }^{12}$, G. Castellini ${ }^{3,13}$, R. D'Alessandro $^{2,3}$, S. Detti ${ }^{3}$, M. Fasoli ${ }^{14,15}$, N. Finetti ${ }^{3,16}$, A. Italiano ${ }^{6}$, P. Lenzi ${ }^{3}$, P. Maestro ${ }^{9,10}$, P.S. Marrocchesi ${ }^{9,10}$, M. Miritello ${ }^{17}$, N. Mori' ${ }^{2,3}$, M. Olmi 2,3 , G. Orzan ${ }^{11}$, L. Pacini ${ }^{2,3,13}$, P. Papini ${ }^{3}$, M.G. Pellegriti ${ }^{6}$, F. Pirzio ${ }^{1,4}$, S. Ricciarini ${ }^{3,13}$, P. Spillantini ${ }^{2}$, O. Starodubtsev ${ }^{3}$, F. Stolzi ${ }^{9,10}$, J.E. Suh ${ }^{9,10}$, A. Sulaj $^{9,10}$,

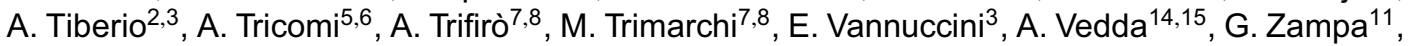 and N. Zampa ${ }^{11}$

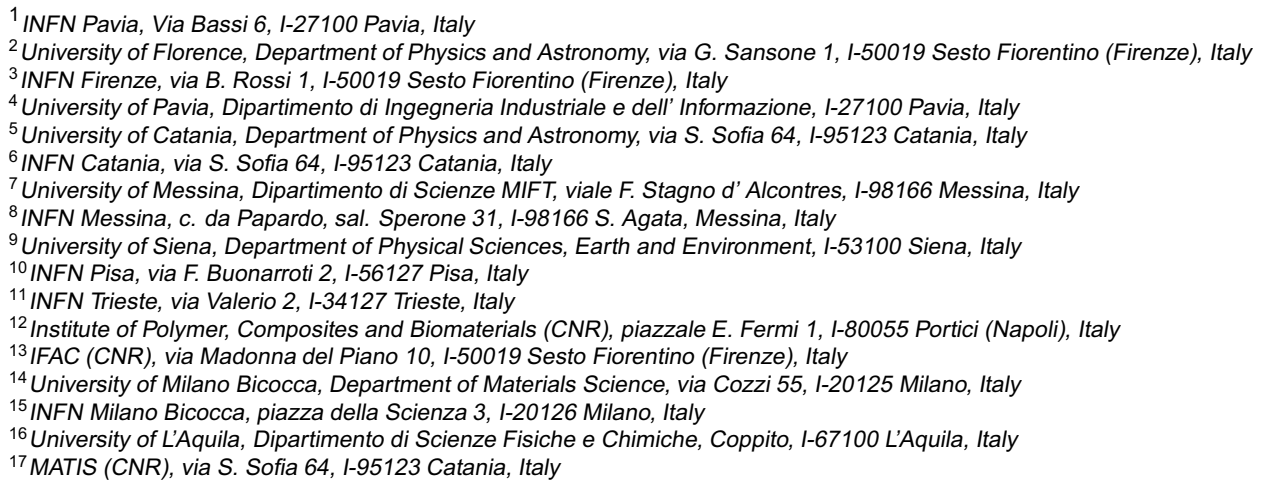

\begin{abstract}
CaloCube is an $\mathrm{R} \& \mathrm{D}$ project borne to develop a novel calorimeter design, optimized for high-energy cosmic ray measurements in space. A small prototype made of $\mathrm{CsI}(\mathrm{Tl})$ elements has been built and tested on particle beams. A final version, made of $5 \times 5 \times 18$ crystals and with dual readout (two photodiodes for each crystal), to cover the full required dynamic range, is under construction and will be tested at CERN SPS in Summer 2016. The dual readout compensation technique were developed and the feasibility to extract Čerenkov signals from CsI crystals verified.
\end{abstract}

\section{Introduction}

The study of cosmic rays can be done either through indirect or direct experiments. The former are commonly achieved by means of ground-based detectors, which detect the secondary particles produced by cosmic rays of very high energy $(\mathrm{E} \geq 1 \mathrm{PeV})$ interacting with the Earth's atmosphere,

\footnotetext{
a e-mail: andrea.rappoldi@pv.infn.it

be-mail: paolo.cattaneo@pv.infn.it
} 
taking advantage of the multiplication effect due to the shower development. On the contrary, the direct measurement concerns the individual detection of the primary cosmic rays of energy up to $10^{15}$ $\mathrm{eV}$ (given the rapid attenuation of the flux with increasing energy), which is of great interest in the study of cosmic rays produced by galactic sources.

This type of measurement is done using instruments placed abovee the atmosphere (on board of orbiting satellites), and represents a significant technological challenge, because such detectors must have: large geometrical acceptance, high energy resolution and optimal particle identification capability, with a limited weight and low power consumption.

\section{The CaloCube concept}

The direct measurement of individual protons and nuclei spectra in space at high energy (up to 10 $\mathrm{PeV}$ ) requires to have: an extremely large acceptance (few $\mathrm{m}^{2} \mathrm{sr}$ ), a good energy resolution (better than $40 \%$ ) and mass identification capability, whereas the direct measurement of the electron component above $10 \mathrm{TeV}$ requires: an excellent energy resolution (better than $2 \%$ ), high $\mathrm{h} / \mathrm{e}$ rejection (better than $10^{5}$ ) and large acceptance above $1 \mathrm{TeV}$. Such stringent requirements can be obtained using a calorimeter in conjunction with a $\mathrm{dE} / \mathrm{dx}$ detector, and for this purpose the $\mathrm{R} \& \mathrm{D}$ project CaloCube ${ }^{1}$ was created, with the aim of studying the design and optimization of a calorimeter for measurements of high-energy cosmic rays in space[1]. The proposed solution consists of a finely segmented calorimeter made of

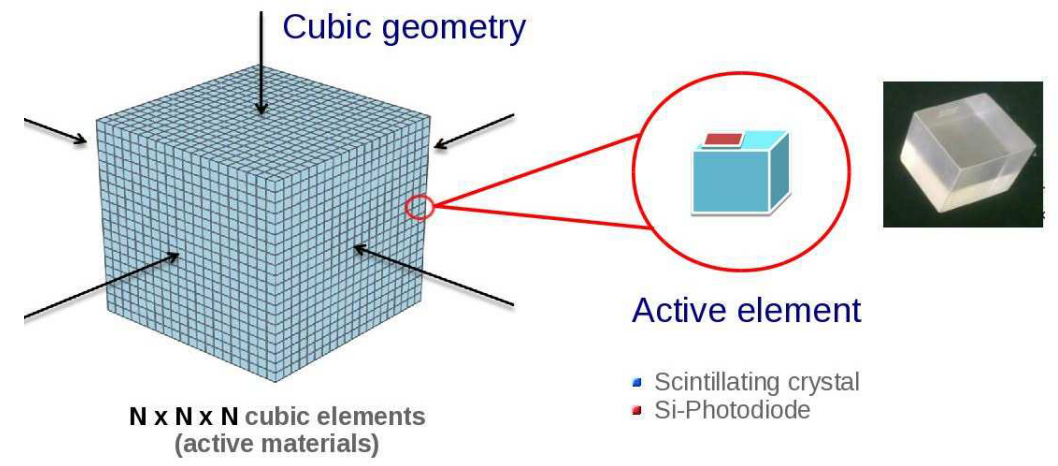

Figure 1. The CaloCube layout is based on a quasi-isotropic geometry with cubic symmetry, where the active volume is made of $\mathrm{NxNxN}$ small cubic scintillator crystals, optically isolated from each other, read from one or more solid state detectors (Si-Photodiode).

a large number of active scintillating elements, arranged in a geometry with cubic symmetry in order to have a quasi-isotropic response (see Fig. 1). Each active element, acting as an independent scintillator, is optically isolated from each other, and is read by one or more silicon photodiode (to have a larger dynamic range). The scintillating crystals also work as active absorber (concerning the energy deposition), resulting in a good energy resolution. Moreover, the 3D segmentation of the calorimeter allows to obtain a precise shower imaging[2].

The calorimeter response depends on the adopted geometry and on the material used for the single scintillating crystals. For this reason, an accurate Montecarlo, based on the FLUKA package, has been developed to carefully study the calorimeter operation in different configurations.

A NxNxN cubic geometry, with cubes of about 1 Molière radius size each, has been studied with different scintillating materials, sizes and gaps (distance between the adjacent cubes). Some re-

\footnotetext{
${ }^{1}$ CaloCube is an R\&D project financed by INFN for 3 years (end 2016).
} 
sults are summarized in Table 2, where different configurations, comparable in terms of total weight ( $W_{\text {tot }} \simeq 2$ tons) and active volume fraction (about 78\%) are shown. Among the five materials, LYSO

\begin{tabular}{|c|c|c|c|c|c|}
\hline & CsI(TI) & BaF $_{\mathbf{2}}$ & YAP(Yb) & BGO & LYSO(Ce) \\
\hline$l(\mathrm{~cm})$ & 3.60 & 3.20 & 2.40 & 2.30 & 2.10 \\
Gap $(\mathrm{cm})$ & 0.30 & 0.27 & 0.20 & 0.19 & 0.18 \\
$N$ cubes & $20 \times 20 \times 20$ & $22 \times 22 \times 22$ & $28 \times 28 \times 28$ & $27 \times 27 \times 27$ & $30 \times 30 \times 30$ \\
$L(\mathrm{~cm})$ & 78.0 & 76.3 & 72.8 & 67.2 & 68.2 \\
$L_{\text {int }}\left(\lambda_{\text {int }}\right)$ & 1.80 & 2.31 & 3.09 & 2.72 & 3.01 \\
$L_{\text {rad }}\left(X_{0}\right)$ & 38.88 & 34.73 & 24.96 & 55.54 & 53.75 \\
$G F\left(\mathrm{~m}^{2} \mathrm{sr}\right)$ & 9.56 & 9.15 & 8.32 & 7.10 & 7.35 \\
\hline
\end{tabular}

Table 1. List of some of the studied calorimeter geometry. Each configuration has been simulated with a FLUKA based Montecarlo, in order to find the best behavior of the calorimeter, having fixed some global parameters, such as the total weight $\mathrm{W}_{\text {tot }} \simeq 2$ tons.

$\left(\mathrm{Lu}_{1.8} \mathrm{Y}_{0.2} \mathrm{SiO}_{5}(\mathrm{Ce})\right)$ is the best one for protons, due to the better shower containment, which compensates for the smaller volume (due to its high density of $7.1 \mathrm{~g} / \mathrm{cm}^{3}$ ).

The collection efficiency of the scintillation light depends on the type of coating used to reflect the light and isolate each crystal from the adjacent ones. Several different materials have been tested measuring the signal induced by a $5.5 \mathrm{MeV} \alpha$ emitted by a ${ }^{241} \mathrm{Am}$ source, and the result (in terms of signal amplitude) is shown in Fig. 2.

\section{Test and results}

A small mechanical structure has been built, to allow the test of different geometric configurations on an accelerator beam. In particular, a prototype with 135 cubic crystals of $3.6 \mathrm{~cm}$ size, arranged in 15 planes of $3 \times 3$ cubes each, with a gap of $0.4 \mathrm{~cm}$ between them, has been implemented (Fig. 3 ). In this case, $\mathrm{CsI}(\mathrm{Tl})$ has been chosen for practical reasons: it is widely available on on the market at an affordable price, it has a very high light yield and its emission spectrum matches very well the spectral response of a large variety of Si photodetectors.

This prototype results to have a shower containment of about 1.5 Molière radius $\left(R_{M}\right)$ and an active depth of $28.4 X_{0}$ and $1.35 \lambda_{\mathrm{int}}$, and has been tested at CERN with different particle beams, summarized in Table 3.

\begin{tabular}{|l|l|l|}
\hline Test & Beam & Energy \\
\hline Feb. 2013 & ions $\mathrm{Pb}+\mathrm{Be}$ & $13-30 \mathrm{GeV}$ \\
Mar. 2015 & Ions Ar + Poly & $19-30 \mathrm{GeV}$ \\
Aug./Sep. 2015 & $\mu, \pi, \mathrm{e}$ & $50,75,150,180 \mathrm{GeV}$ \\
\hline
\end{tabular}

During the last test beam at $\operatorname{CERN}^{2}$ (Summer 2015), the prototype was initially exposed to $\mu$ beams, to obtain the response of each crystal to a minimum ionizing particle (m.i.p.), thus determining the individual conversion factor between the deposited energy and the photodiode signal (Fig. 4). These factors are used to equalize the response of all the cubes that compose the calorimeter.

Then an estimate of the energy resolution has been determined, exposing the calorimeter to $\mathrm{e}^{+} / \mathrm{e}^{-}$ beams of different energy, and determining the total deposited energy, given by the sum of the equalized signals of all the cubes. A preliminary result is shown in Fig. 5, referring to a beam of $50 \mathrm{GeV}$ electrons. In this case the measured total energy (expressed in m.i.p) is in good agreement with the expectation, and the corresponding resolution is at level of $1 \%$.

2 AIDA ${ }^{2020}$ This project has received funding from the European Union's Horizon 2020 research and innovation programme under grant agreement No 654168 


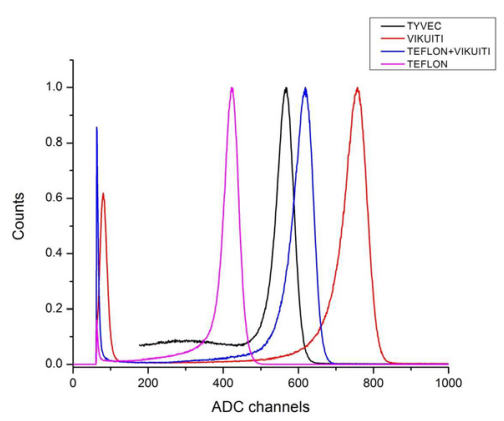

Figure 2. Signal amplitude of a single CsI(T1) scintillator cubic crystal of size $(36 \mathrm{~mm})^{2}$ with different coating materials.

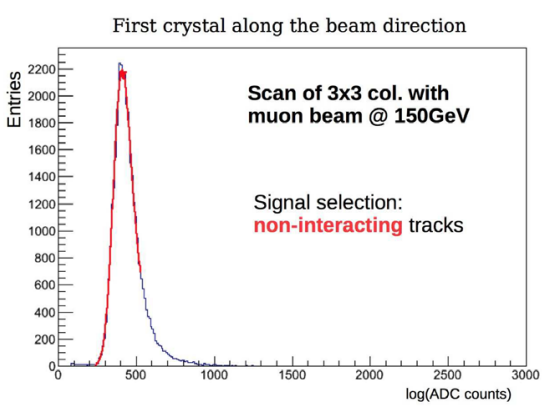

Figure 4. Response of a single crystal to a noninteracting track at $150 \mathrm{GeV}$ (mainly $\mu$ ). The distribution is well fit with a Landau distribution (red).

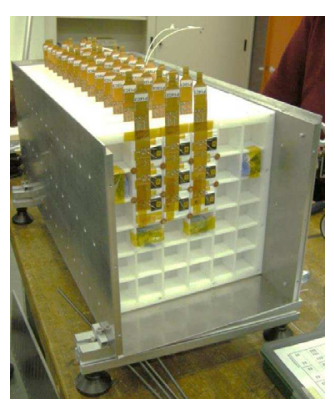

Figure 3. The prototype with $3 \times 3 \times 15 \mathrm{CsI}(\mathrm{Tl})$ crystals used to perform the test beams at CERN in 2015.

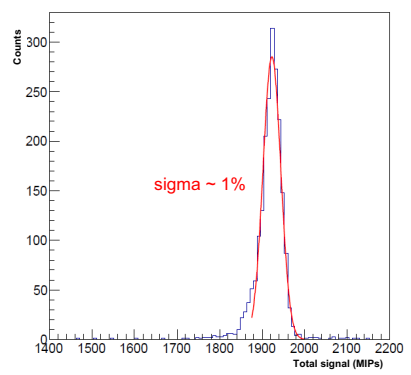

Figure 5. Measured distribution of total energy (expressed in m.i.p.) released with a $50 \mathrm{GeV}$ electrons beam, fit with with the expected distribution (red). The resulting energy resolution is about $1 \%$.

\section{Next developments}

A new calorimeter prototype, consisting of $5 \times 5 \times 15$ cubes, is in preparation and under test at CERN in the summer 2016. The larger number of cubes (especially in the transverse dimension) allows to have a better event containment, even for hadronic showers. In this prototype the light signal of each crystal will be read out by two photodiodes, with different sensitive area, in order to cover the full expected dynamic range.

Furthermore, some tests are in progress to verify the feasibility of detecting also the Čerenkov light produced by charged particles inside the crystals (CsI is transparent down to $340 \mathrm{~nm}$ wavelength), to increase the calorimeter performance. The first results demonstrate that the separation between the scintillation and Čerenkov light can be obtained relying both on timing (prompt vs. delayed signal) and on wavelength (UV vs. green).

\section{References}

[1] M. Bongi et al., J. Phys. Conf. Ser. 587, 012029 (2015)

[2] R. D’Alessandro et al., Nucl. Instrum. Meth. A824, 609 (2016) 